\title{
Investigation of Interaction Between Physiological Signals and fMRI Dynamic Functional Connectivity using Independent Component Analysis*
}

\author{
F. Nikolaou, C. Orphanidou, K. Murphy, R. G. Wise and G. D. Mitsis
}

\begin{abstract}
The blood oxygen level dependent (BOLD) fMRI signal is influenced not only by neuronal activity but also by fluctuations in physiological signals, including respiration, arterial $\mathrm{CO} 2$ and heart rate/ heart rate variability (HR/HRV). Even spontaneous physiological signal fluctuations have been shown to influence the BOLD fMRI signal in a regionally specific manner. Consequently, estimates of functional connectivity between different brain regions, performed when the subject is at rest, may be confounded by the effects of physiological signal fluctuations. In addition, resting functional connectivity has been shown to vary with respect to time (dynamic functional connectivity - DFC), with the sources of this variation not fully elucidated. The effect of physiological factors on dynamic (timevarying) resting-state functional connectivity has not been studied extensively, to our knowledge. In our previous study, we investigated the effect of heart rate $(\mathrm{HR})$ and end-tidal $\mathrm{CO} 2$ (PETCO2) on the time-varying network degree of three welldescribed RSNs (DMN, SMN and Visual Network) using maskbased and seed-based analysis, and we identified brain-heart interactions which were more pronounced in specific frequency bands. Here, we extend this work, by estimating DFC and its corresponding network degree for the RSNs, employing a datadriven approach to extract the RSNs (low- and high-dimensional Independent Component Analysis (ICA)), which we subsequently correlate with the characteristics of simultaneously collected physiological signals. The results confirm that physiological signals have a modulatory effect on resting-state, fMRI-based DFC.
\end{abstract}

\section{INTRODUCTION}

Functional Connectivity analysis, i.e. investigating the correlation of neuroimaging signals between different brain regions [1,2], has gained a lot of popularity in the past few years as a tool for advancing our understanding of the synchronized variations in the neuronal activity of a network of brain regions. Even in the case of Resting State (RS) fMRI, intensity have been shown to be correlated between

* Research supported by ABC Foundation.

F. Nikolaou is with the KIOS Research and Innovation Center of Excellence and Department of Electrical and Computer Engineering in the University of Cyprus (corresponding author to provide phone: +35799114481; e-mail: nikolaou.foivia@ucy.ac.cy)

C. Orphanidou is with the Department of Electrical and Computer Engineering in the University of Cyprus (email: orphanid@ucy.ac.cy)

$\mathrm{K}$. Murphy is with Cardiff University Brain Research Imaging Center (CUBRIC), School of Psychology, Cardiff University (email: murphyk2@cardiff.ac.uk)

R. G. Wise is with Cardiff University Brain Research Imaging Center (CUBRIC), School of Psychology, Cardiff University (email: WiseRG@ cardiff.ac.uk)

G. D. Mitsis is with Department of Bioengineering, McGill University (email: georgios.mitsis@mcgill.ca) functionally related areas. Related to RS fMRI studies, where the signal modulations are measured in the absence of a task, low frequency $(<0.1 \mathrm{~Hz})$ fluctuations in the fMRI signal recent discovery that spontaneous brain activity is not random noise but is particularly organized in resting state networks (RSNs) has created significant interest, as RSNs provide a way to probe brain function without needing explicit tasks to drive brain activity $[3,4]$. Several studies have identified numerous consistent and distinct RSNs, including motor, auditory, visual, and attention networks [5,6], as well as the presence of RSNs using additional modalities such as electroencephalography and magnetoencephalography $[7,8]$.

RSN studies are, however, hindered by the presence of non-stationarities which are directly attributable to neuronal activity. Besides neuronal fluctuations, a possible source of these non-stationarities is the fact that the fMRI blood-oxygen level dependent (BOLD) signal is also modulated by nonneuronal physiological variability (e.g. heart rate, arterial $\mathrm{CO} 2$, respiration). The impact of these variables on fMRI measurements and connectivity has been established [9-10]. More specifically, the cerebral vasculature has been shown to be exquisitely sensitive to arterial $\mathrm{CO} 2$ fluctuations: spontaneous arterial $\mathrm{CO} 2$ fluctuations affect both cerebral blood flow in the middle cerebral artery [11] and the fMRI BOLD signal regionally [9], [12]. Furthermore, slow changes in respiration depth and rate were found to significantly affect resting-state functional connectivity of the DMN [13] and $\mathrm{HRV}$, as well as its low frequency and high frequency power components, have been shown to be correlated to regional BOLD signal variations [10]. RSN studies are particularly susceptible to the effect of physiological noise, since they are usually based on a single experimental trial [14-15].

The effect of physiological factors on resting-state DFC has not been studied extensively, to our knowledge. In our previous study $[16,17]$, we investigated the effect of heart rate (HR) and end-tidal CO2 (PETCO2) on the time-varying network degree of three well-described RSNs and identified brain-heart interactions which were more pronounced in specific frequency bands using mask-based and seed-based analysis. Those approaches require some prior knowledge/assumptions for defining the corresponding regions of interest (ROI) and may, thus, introduce a bias to the results. To address this, in the present paper we extend this work by estimating DFC and its corresponding network degree for the DMN, visual and somatosensory RSNs with a datadriven approach. Specifically, we use low- and highdimensional Independent Component Analysis (ICA) to extract the RSNs, the properties of which we subsequently 
correlate with the simultaneously collected physiological signals. Moreover, the effect of respiration per volume (RVT) was included, in addition to HR and PETCO2.

\section{MATERIALS AND METHODS}

\section{A. Experimental Data}

The experimental data were collected at the Cardiff University Brain Imaging Centre (CUBRIC), where twelve healthy volunteers ( 7 male, aged $29.2 \pm 4.6$ years) underwent resting-state fMRI. A 3T scanner (General Electric) was used to perform the imaging. Head movement was minimized with a bite bar. For each subject, a T1-weighted FSGPR structural scan $(256 \times 256 \times 172$ voxels of $1 \times 1 \times 1 \mathrm{~mm})$ was acquired and used to assist in placing individual subjects' data into a common stereotactic space. During the resting-state scan, the subjects were instructed to keep their eyes closed and not to fall asleep. The duration of the experiment was 630sec, corresponding to 210time-points (gradient echo EPI sequence, $\mathrm{TR}=3 \mathrm{sec}, \mathrm{TE}=35 \mathrm{~ms}, \mathrm{FOV} / \mathrm{slice}=20.5 \mathrm{~cm} / 3.2 \mathrm{~mm}$, flip $=90^{\circ}, 53$ slices with $91 \times 109 \times 91$ voxels of $3.2 \times 3.2 \times 3.2 \mathrm{~mm}$ ). PETCO2 signal, RVT signal and ECG waveforms were recorded in parallel to the scanning experiment.

\section{B. fMRI and Physiological Data Pre-processing}

A processing pipeline was applied which was developed using FSL [18] and in-house-developed MATLAB scripts. The pre-processing of the experimental fMRI data consisted of various steps such as the skull removal for functional images using the BET function.

PETCO2 and ECG signals were recorded in parallel to the scanning experiment. PETCO2 was recorded using a nasal cannula attached to a gas analyser (AEI Technologies). ECG waveforms obtained during the scan were used to extract the HR and HRV signals. Firstly, the raw signal quality was checked by using a Signal Quality Index (SQI) which labels every ten seconds of data as "reliable" or "unreliable", based on a set of physiologically-relevant checks, followed by QRS template matching [19]. For the subsequent computation and analysis, only time series which were labeled as "reliable" for more than $80 \%$ of the time were used. Subsequently, QRS detection was performed using the Hamilton and Tompkins algorithm [20]. The HRV signal at each beat was defined as the periods between consecutive R-peaks, while the HR signal was defined as the inverse of these periods multiplied by $60-$ in units of beats/min. Spline interpolation at a frequency of 4 $\mathrm{Hz}$ (to account for the subsequent extraction of $\mathrm{HF}$ frequencies) was applied to obtain the final HR and HRV signals and subsequently filtering with a median filter in order to remove spurious spike artefacts. During the experiment, the volunteers during the fMRI duration wore 3 respiration belts in order to compare the position of different belts. For this study, only the inverted respiration signal was used.

\section{Resting-state Dynamic Functional Connectivity Analysis \\ 1) Independent Component Analysis}

ICA analysis was carried out using FSL 5.0.9 MELODIC, whereby the three first volumes were regressed out from the analysis. The single-subject ICA was performed conventionally, using the entire timeseries consisting of all 210 brain volumes imaged [21]. In this ICA approach, two time-sliding window analyses were applied by using a length of 50 time lags (as determined in our previous study [16, 17]) and a 1 or 10 time lag increment respectively. For each RSN of interest (DMN, visual and somatosensory), the average BOLD fMRI time series in each area within the RSN was correlated with the others in order to obtain a functional connectivity matrix within each window. Specifically, the cross-correlation between two time-series is estimated from finite data samples using the following sum:

$$
\widehat{\Phi}_{x y}(m)=\frac{1}{N-|m|} \sum_{n=0}^{N-|m|-1} y(n+m) x(n)
$$

where $x, y$ are the two time series (in our case, fMRI time series corresponding to different regions), $N$ is data length and $m$ is cross-correlation lag. After obtaining cross-correlation values (both the absolute maximum and the absolute average cross-correlation values within \pm 5lags were calculated) between all areas in the examined RSNs, the overall timevarying connectivity of the network was quantified by computing the graded network degree within each window. Specifically, the graded degree is defined as:

$$
k_{i}^{W}=\sum_{j \in M} W_{i j}
$$

where $\mathrm{M}$ is the number of brain regions in the network and $\mathrm{W}_{\mathrm{ij}}$ are the absolute maximum (or average) correlation values (within \pm lags) between the network nodes $i$ and $j$., whereby each node corresponds to a different region.

The power spectral density of the physiological signals was calculated within the same sliding windows by applying the Welch method [22]. The values of the graded network degree in each window were subsequently correlated to the time-varying power spectral density of the examined physiological signals.

\section{Low Dimensionality ICA:}

For the low-dimensionality ICA, a model order of 25 ICs was chosen, in line with previous studies and guidelines [23]. In general, using model orders around 20 provides a general picture of large scale brain networks. However, detection of some network components (i.e., S1, S2 (which are parts of the $\mathrm{SMN}$ ), and striatum) requires higher model order estimation. Model orders between 30-40 have shown spatial overlapping of some IC sources [23]. The 25 ICs were first manually classified as RSNs or motion-related ICA components. To do this, the spatial map, time course and power spectrum of the time course for each component were used. Subsequently, ICA-AROMA, which is a robust ICA-based strategy for removing motion artifacts from fMRI data was used [24].

\section{High Dimensionality ICA:}

For the high-dimensionality ICA, a dimensionality of 70 ICs was selected based on previous studies [23] showed that ICA analyses results are affected by model order selection. In addition, another study demonstrated that, in patients with seasonal affected disorder, the between-group differences measured with ICA increased with model order (reaching a maximum around 70 components) [25]. These 70 components were subsequently classified by taking the low-dimensional RSNs as reference templates and using a spatio-temporal labeling criterion: the high dimensional component $i$ was 
labeled as part of the low-dimensional component $j$ with which it had the highest spatial overlap (using the fslcc function in FSL) as well as the highest temporal correlation (calculated with Spearman's correlation among single-subject time series). A component was classified as residual noise if all spatial overlap and temporal correlations were below a threshold empirically determined during the algorithm development by evaluating different values against manual classification (0.4 for spatial overlap and 0.4 for temporal correlation). Both the residual noise and the components form other networks or areas of the brain except the DMN, visual and somatosensory networks were ignored in subsequent analyses.

\section{2) Wavelet Transformation}

All the signals were interpolated at $4 \mathrm{~Hz}$ to be consistent and a 7-level wavelet decomposition was applied to the physiological signals (Figure 1) in order to obtain the corresponding frequency decompositions and calculate correlations with the corresponding time-varying RSN degree within all the resulting frequency sub-bands. Note that the choice of wavelet basis function did not have a significant effect on our analysis. The results presented were obtained by using the Coiflets wavelet basis [26].

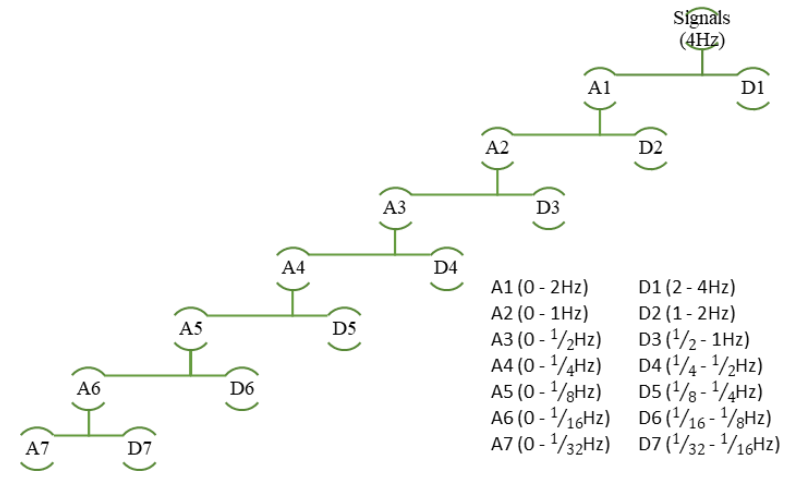

Figure 1. A 7-level wavelet decomposition was applied to all physiological signals

\section{3) Correlation Coefficients}

In all the cases, the modulating effect of time-varying total and band-limited power for all physiological signals (PETCO2, RVT, HR and HRV) on the time-varying RSN degree was quantified by calculating the Spearman rank correlation coefficient, which is suitable in our case due to the fact that our results are paired with respect to time (network degree versus time-varying physiological signal power). The analysis was performed on all 12 subjects (11 for HR analysis, as one subject did not satisfy the SQI mentioned above) and the mean and standard deviation of the resulting correlation coefficients over all subjects were calculated.

\section{REsults}

\section{A. Resting State Dynamic Functional Connectivity Analysis}

1) Low Dimensionality ICA

For the low dimensionality analysis, out of the 25 independent components, 9 RSNs were identified. Our analysis was then focused on the DMN, the somatosensory network (SMN) and the visual network (see Figure 2).

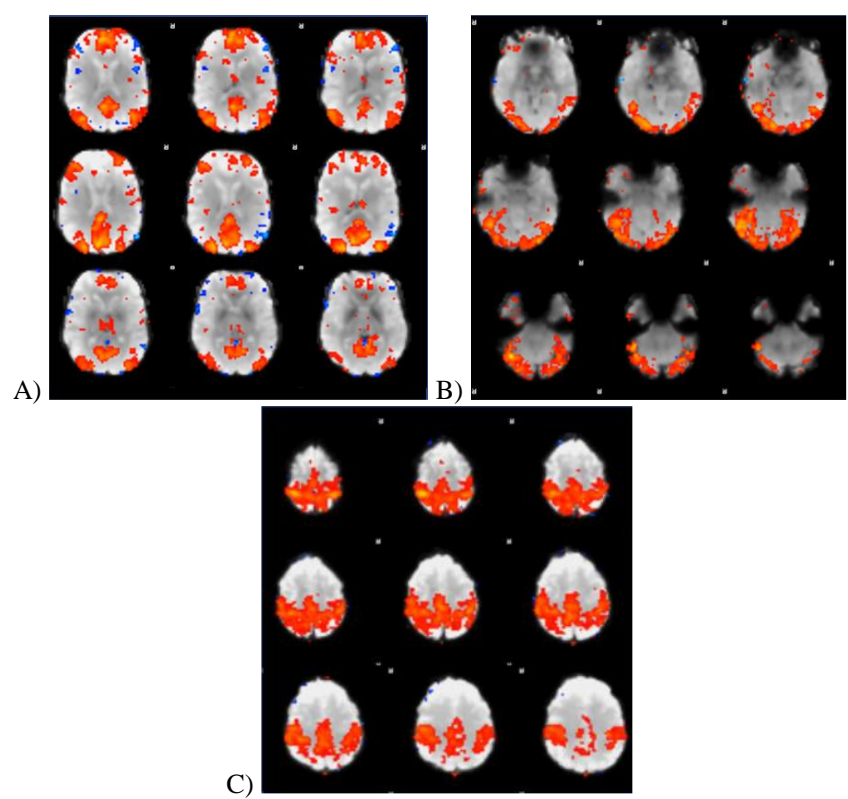

Figure 2. Low dimensional components relative to the networks of interest selected for this study: A) the DMN, B) the visual network and C) the SMN

The Spearman correlation coefficients between timevarying DMN/SMN/Visual network degree and band-limited PETCO2/RVT/HR/HRV power for the 10 and 1 time lag sliding window increments are given in Table I for the subbands of maximum correlation.

\begin{tabular}{|c|c|c|c|c|c|}
\hline \multicolumn{6}{|c|}{ LOW DIMENSIONALITY } \\
\hline & & \multicolumn{2}{|c|}{$\begin{array}{l}10 \text { TIME-POINT } \\
\text { INCREMENT }\end{array}$} & \multicolumn{2}{|c|}{$\begin{array}{l}1 \text { TIME-POINT } \\
\text { INCREMENT }\end{array}$} \\
\hline \multirow{5}{*}{ DMN } & & MEAN & STD & MEAN & STD \\
\hline & CO2-A6 & 0.5321 & 0.1 & 0.5844 & 0.13 \\
\hline & RVT-A6 & 0.5607 & 0.05 & 0.6032 & 0.083 \\
\hline & HR-A4 & 0.6179 & 0.08 & 0.6755 & 0.11 \\
\hline & HRV-A4 & 0.5821 & 0.079 & 0.6655 & 0.11 \\
\hline \multirow{4}{*}{ SMN } & CO2-A7 & 0.5357 & 0.15 & 0.5571 & 0.18 \\
\hline & RVT-A7 & 0.5214 & 0.09 & 0.5571 & 0.12 \\
\hline & HR-A5 & 0.6143 & 0.075 & 0.6092 & 0.10 \\
\hline & HRV-A5 & 0.575 & 0.072 & 0.5999 & 0.11 \\
\hline \multirow{4}{*}{ VISUAL } & CO2-A8 & 0.5679 & 0.13 & 0.5231 & 0.16 \\
\hline & RVT-A8 & 0.6532 & 0.12 & 0.6532 & 0.15 \\
\hline & HR-A6 & 0.7286 & 0.077 & 0.8118 & 0.10 \\
\hline & HRV-A6 & 0.7932 & 0.089 & 0.7964 & 0.12 \\
\hline
\end{tabular}

Table I. Low Dimensionality ICA: Spearman correlation coefficients between time-varying DMN/SMN/Visual network degree and timevarying PETCO2/RVT/HR/HRV band-limited signal power for 10 and 1 time-lag sliding window increments. CO2-A6: wavelet level A6 (0-0.063Hz), RVT-A6: wavelet level A6(0-0.063Hz), HR-A4: wavelet level A4 (0-0.25 Hz), HRV-A4: wavelet level A4 $(0-0.25 \mathrm{~Hz})$.

These suggest the presence of temporal correlations between network degree and the band-limited power of the physiological signals. In more detail, the wavelet level A6 yielded higher values for the PETCO2 and RVT signals, whereas the wavelet level A4 yielded higher values for HR and HRV. The values obtained for HR and HRV were, as expected, very similar. The sliding window approach using 
one time-lag increment yielded slightly higher values compared to the 10 time-lag increments.

\section{2) High Dimensionality ICA}

Components of interest, related to the DMN, Visual network and SMN (see Figure 3) were apparent in several subcomponents when high dimensionality ICA was performed. Out of the 70 independent components, we identified 47 sub-networks of RSNs and detected 22 RSNs in total, including the DMN, SMN, Visual, Auditory and Basal Ganglia networks. Both the total number of RSNs and the number of RSN sub-networks increased as a function of ICA model order. Specifically, in the low dimensionality ICA only 9 RSNs were identified.

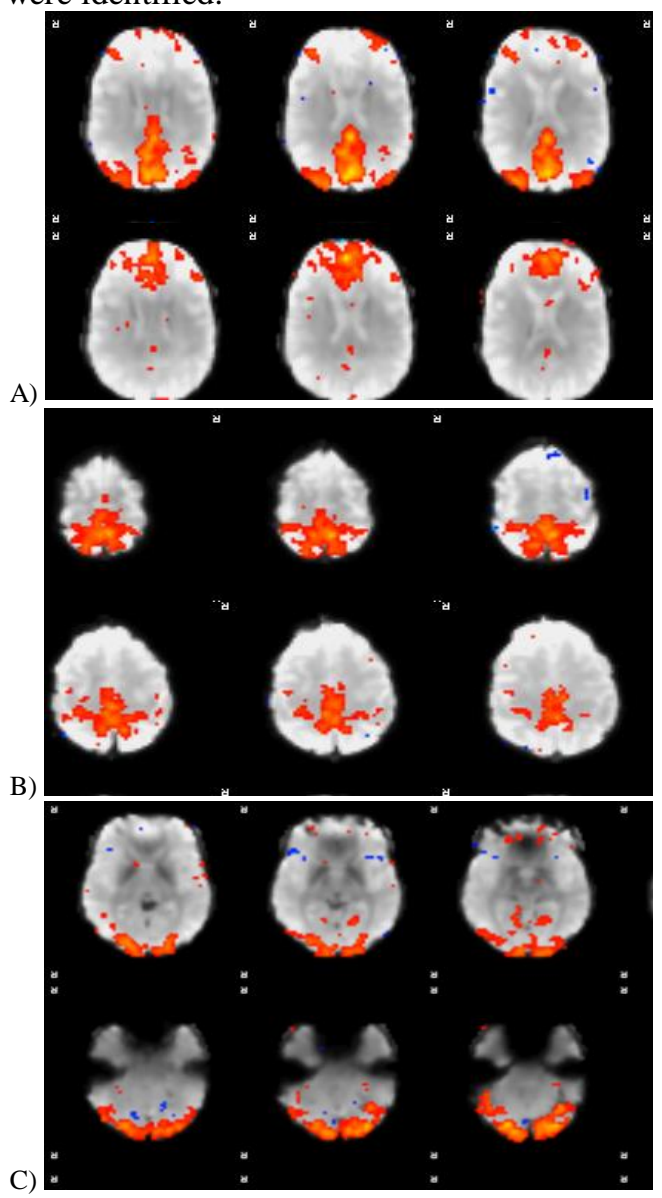

Figure 3. High dimensional components relative to the networks of interest selected for this study: A) the DMN, B) the visual network and $C$ ) the $S M N$

The same procedure used for the low dimensionality ICA for the three mentioned RSNs (DMN, Visual network and SMN) was applied for the high dimensionality ICA. The band-limited power of all physiological signals (PETCO2, RVT, HR, HRV) was used to obtain the subsequent results. The Spearman correlation coefficients for the high dimensionality ICA between time-varying DMN/SMN/ Visual network degree and band-limited PETCO2/RVT/ HR/HRV power for the 10 and 1 time-lag increments are given in Table II. The correlations coefficients are averaged over all subjects (mean \pm standard deviation).

\section{HIGH DIMENSIONALITY}

10 TIME-POINT 1 TIME-POINT

INCREMENT INCREMENT

\begin{tabular}{llcccc}
\hline & & MEAN & STD & MEAN & STD \\
& CO2-A6 & 0.6505 & 0.20 & 0.6571 & 0.22 \\
DMN & RVT-A6 & 0.6705 & 0.15 & 0.6771 & 0.17 \\
& HR-A4 & 0.8 & 0.18 & 0.8179 & 0.20 \\
& HRV-A4 & 0.7829 & 0.18 & 0.8144 & 0.20 \\
\hline \multirow{3}{*}{ SMN } & CO2-A7 & 0.5821 & 0.25 & 0.5839 & 0.27 \\
& RVT-A7 & 0.5929 & 0.19 & 0.6486 & 0.21 \\
& HR-A5 & 0.6429 & 0.17 & 0.641 & 0.19 \\
& HRV-A5 & 0.6286 & 0.17 & 0.6497 & 0.19 \\
\hline \multirow{2}{*}{ VISUAL } & CO2-A8 & 0.5964 & 0.23 & 0.6214 & 0.25 \\
& HRT-A8 & 0.6946 & 0.22 & 0.7071 & 0.25 \\
& HRV-A6 & 0.7794 & 0.18 & 0.8179 & 0.20 \\
\hline
\end{tabular}

Table II. High Dimensionality ICA: Spearman correlation coefficients between time-varying DMN/SMN/Visual network degree and time-varying PETCO2/RVT/HR/HRV band-limited signal power for 10 and 1 time-point increments. CO2-A6: wavelet level A6 (O$0.063 \mathrm{~Hz}), \mathrm{RVT}-\mathrm{A6}$ : wavelet level $\mathrm{A6}(0-0.063 \mathrm{~Hz}), \mathrm{HR}-\mathrm{A} 4$ : wavelet level A4 (0-0.25 Hz), HRV-A4: wavelet level A4 (0-0.25 Hz).

As can be seen from the above tables, results were overall in agreement with the low-dimensional ICA. As before, our results suggest the presence of temporal correlations between network degree and the band-limited power of the physiological signals. The wavelet level A6 $(0-0.063 \mathrm{~Hz})$ yielded the highest values for the PETCO2 and RVT signals, whereas the wavelet level A4 $(0-0.25 \mathrm{~Hz})$ yielded higher values for the HR and HRV. The sliding window using one time-point offset yielded slightly higher values against the 10 time-points offset.

\section{DISCUSSION}

The present study examines the modulation of dynamic, resting-state connectivity by physiological signal fluctuations. The results reveal a clear effect of time-varying signal power for PETCO2, RVT, HR and HRV on the time-varying network degree for three well-described RSNs: the default-mode, visual and somatosensory RSNs, revealing brain-heart interactions in the context of fMRI-based RSN connectivity studies. This effect was found to be more pronounced for the fluctuations in the physiological spectral content in specific frequency sub-bands (time-varying band-limited power), as revealed by wavelet analysis. Despite the well-established effect of physiological signals on fMRI connectivity and particular RSN connectivity [9,10], [13-15], the effect of timevarying properties of physiological signals on resting-state DFC has not been examined to a large extent. The significance of this is that it suggests that even moderate modulations in the power of these signals can considerably influence RSN analyses and that a significant source of dynamic variations in resting-state connectivity is physiological in nature. Given that 
resting-state (spontaneous) fluctuations of physiological signals such as PETCO2, RVT, HR and HRV are of small magnitude, they are not expected to significantly effect neuronal activity per se; therefore, the observed modulatory effects are likely physiological in origin.

ICA analysis was examined to define the RSNs of interest. Low dimensionality ICA was used to identify the most commonly observed RSNs and subsequently high dimensionality ICA was used to investigate subnetworks inside each RSN. The high dimensionality ICA yielded a larger number of components within each RSN, which were used to calculate the network degree compared to the low dimensionality approach. ICA model order selection significantly influenced the RSN characteristics. At high ICA model orders, a network may be 'split' into a number of subnetworks depending on the number of estimated ICA components. Thus, the choice of ICA model order is a crucial element in the analysis, especially in functional brain connectivity studies. Large-scale networks (i.e. low model order components) are compact and easy to identify and include networks such as the visual, auditory, sensory-motor, etc. At low model orders (e.g. < 25), signal sources tend to aggregate into singular components involving various neuroanatomically and functionally separate units. These units become detectable later as separate components at higher model orders.

Our results show that the wavelet decomposition yields considerably stronger correlations between network degree and physiological signal power, in agreement to our previous study using mask and seed-based analysis [16, 17]. Furthermore, the wavelet sub-bands that yielded the strongest correlations between time-varying degree and band-limited power were overall the same throughout our examinations. These sub-bands are related to the spectral characteristics of each signal characteristic and suggest that fluctuations in these bands have a clearer modulatory effect. For PETCO2, modulations in very low frequency power (wavelet level A6 $(0-0.0625 \mathrm{~Hz}))$ were found to have the clearer effect, which is consistent with the previously described spectral characteristics of PETCO2 fluctuations [11]. For RVT, modulations were found to have the clearer effect in the same frequency range with the PETCO2 (wavelet level A6 (0$0.0625 \mathrm{~Hz})$ ). For HR, a wider frequency band was identified (wavelet level A4 $(0-0.25 \mathrm{~Hz})$ ) reflecting the fact that $\mathrm{HR}$ has a richer spectral content than PETCO2. Additionally, the identified frequency band contains both the low frequency (LF - around $0.04-0.15 \mathrm{~Hz}$ ) spectral peak and possibly also the high frequency $(\mathrm{HF}$ - around $0.15-0.4 \mathrm{~Hz})$ spectral peak of the HR signal. While, often, these two spectral peaks are assumed to correspond to cardiac sympathetic and cardiac parasympathetic neural activity, respectively, the relative contributions of each mechanism still remain the subject of controversy and investigation.

Finally, for the low and high dimensionality ICA analysis and sliding window analysis with a 1 time-lag increment, all the limited bands of the power of the physiological signals yielded the highest correlation values. This sliding window analysis helped to assess the DFC with a better resolution.

\section{CONCLUSION}

The dynamic association between functional connectivity and physiological signals in the resting state have been investigated using both ICA to define the RSNs and slidingwindow analyses of functional connectivity. The finding of a significant correlation between functional connectivity and physiological signals could demonstrate potential factors underlying dynamic changes in resting-state connectivity. Following this, delineating the brain regions exhibiting such connectivity modulation may bring further insight into neural mechanisms underlying autonomic control mechanisms.

\section{REFERENCES}

[1] B. P. Rogers, V. L. Morgan, a T. Newton, and J. C. Gore, “Assessing functional connectivity in the human brain by fMRI," Magn Reson Imaging, vol. 25, no. 1, pp. 1347-1357, 2007.

[2] K. J. Friston, "Functional and effective connectivity: a review.," Brain Connect., vol. 1, no. 1, pp. 13-36, Jan. 2011.

[3] M. D. Fox and M. E. Raichle, "Spontaneous fluctuations in brain activity observed with functional magnetic resonance imaging.," Nat. Rev. Neurosci., vol. 8, no. 9, pp. 700-11, Sep. 2007.

[4] B. Biswal, F. Z. Yetkin, V. M. Haughton, and J. S. Hyde, "Functional connectivity in the motor cortex of resting human brain using echoplanar MRI.," Magn. Reson. Med., vol. 34, no. 4, pp. 537-41, Oct. 1995.

[5] J. S. Damoiseaux, S. A. R. B. Rombouts, F. Barkhof, P. Scheltens, C. J. Stam, S. M. Smith, and C. F. Beckmann, "Consistent resting-state networks," no. 2, 2006.

[6] M. De Luca, C. F. Beckmann, N. De Stefano, P. M. Matthews, and S. M. Smith, "fMRI resting state networks define distinct modes of longdistance interactions in the human brain.," Neuroimage, vol. 29, no. 4, pp. 1359-67, Mar. 2006.

[7] D. J. a. Smit, C. J. Stam, D. Posthuma, D. I. Boomsma, and E. J. C. de Geus, "Heritability of 'small-world' networks in the brain: A graph theoretical analysis of resting-state EEG functional connectivity," Hum. Brain Mapp., vol. 29, no. 12, pp. 1368-1378, 2008.

[8] F. de Pasquale, S. Della Penna, A. Z. Snyder, C. Lewis, D. Mantini, L. Marzetti, P. Belardinelli, L. Ciancetta, V. Pizzella, G. L. Romani, and M. Corbetta, "Temporal dynamics of spontaneous MEG activity in brain networks.," Proc. Natl. Acad. Sci. U. S. A., vol. 107, no. 13, pp. 6040-5, Mar. 2010.

[9] R. G. Wise, K. Ide, M. J. Poulin, and I. Tracey, "Resting fluctuations in arterial carbon dioxide induce significant low frequency variations in BOLD signal.," Neuroimage, vol. 21, no. 4, pp. 1652-64, Apr. 2004.

[10] V. Napadow, R. Dhond, G. Conti, N. Makris, E. N. Brown, and R. Barbieri, "Brain correlates of autonomic modulation: combining heart rate variability with fMRI.," Neuroimage, vol. 42, no. 1, pp. 169-77, Aug. 2008.

[11] G. D. Mitsis, M. J. Poulin, P. A. Robbins, and V. Z. Marmarelis, "Nonlinear modeling of the dynamic effects of arterial pressure and CO 2 variations on cerebral blood flow in healthy humans," IEEE Trans. Biomed. Eng., vol. 51, no. 11, pp. 1932-1943, 2004.

[12] K. T. S. Pattinson, G. D. Mitsis, A. K. Harvey, S. Jbabdi, S. Dirckx, S. D. Mayhew, R. Rogers, I. Tracey, and R. G. Wise, "Determination of the human brainstem respiratory control network and its cortical connections in vivo using functional and structural imaging," Neuroimage, vol. 44, no. 2, pp. 295-305, 2009.

[13] R. M. Birn, J. B. Diamond, M. a. Smith, and P. a. Bandettini, "Separating respiratory-variation-related fluctuations from neuronalactivity-related fluctuations in fMRI," Neuroimage, vol. 31, pp. 15361548, 2006.

[14] K. Murphy, R. M. Birn, and P. a Bandettini, "Resting-state fMRI confounds and cleanup.," Neuroimage, vol. 80, pp. 349-59, Oct. 2013.

[15] R. M. Birn, "The role of physiological noise in resting-state functional connectivity.," Neuroimage, vol. 62, no. 2, pp. 864-70, Aug. 2012. 
[16] F. Nikolaou, C. Orphanidou, P. Papakyriakou, K. Murphy, R.G. Wise and G.D. Mitsis "Spontaneous Physiological Variability Modulates Dynamic Functional Connectivity in Resting-State fMRI," Philosophical Transactions of the Royal Society A 374.2067: 20150183, 2016.

[17] F. Nikolaou, C. Orphanidou, R.G. Wise et al, "Arterial CO2 effects modulate dynamic functional connectivity in resting-state fMRI," Conf Proc IEEE Eng Med Biol Soc., 2015:1809-1812, 2015.

[18] S. M. Smith, M. Jenkinson, M. W. Woolrich, C. F. Beckmann, T. E. Behrens, H. Johansen-Berg, P. R. Bannister, M. De Luca, I. Drobnjak, D. E. Flitney, R. K. Niazy, J. Saunders, J. Vickers, Y. Zhang, N. De Stefano, J. M. Brady, and P. M. Matthews, "Advances in functional and structural MR image analysis and implementation as FSL.," Neuroimage, vol. 23 Suppl 1, pp. S208-19, 2004.

[19] C. Orphanidou, T. Bonnici, P. Charlton, D. Clifton, D. Vallance, and L. Tarassenko, "Signal-quality indices for the electrocardiogram and photoplethysmogram: Derivation and applications to wireless monitoring," IEEE J. Biomed. Heal. Informatics, vol. 19, no. 3, pp. 832-838, 2015.

[20] P. S. Hamilton and W. J. Tompkins, "Quantitative investigation of QRS detection rules using the MIT/BIH arrhythmia database.," IEEE Trans. Biomed. Eng., vol. 33, no. 12, pp. 1157-1165, 1986.

[21] O. Dipasquale, L. Griffanti, M. Clerici, R. Nemni, G. Baselli, F. Baglio, "High-dimensional ICA analysis detects within-network functional connectivity damage of default-mode and sensory-motor networks in Alzheimer's disease," Front Hum Neurosci 9,43, 2015.

[22] A.V. Oppenheim, R.W. Schafer, "Discrete-time signal processing", 3rd edn. Prentice-Hall Signal Processing Series. Englewood Cliffs, NJ: Prentice-Hall, 2009.

[23] A. Abou-Elseoud, T. Starck, J. Remes, J. Nikkinen, O. Tervonen, V. Kiviniemi, "The effect of model order selection in group PICA," Human Brain Mapping 31(8),1207-1216, 2010.

[24] J.X. MI, "A novel algorithm for independent component analysis with reference and methods for its applications," Plos One 9(5), e93984, 2014.

[25] A. Abou Elseoud et al., "Group-ICA model order highlights patterns of functional brain connectivity," Frontiers in Systems Neuroscience $5,37,2011$

[26] I. Daubechies, "Ten lectures on wavelets," In CBMS-NSF regional conference series in applied mathematics, vol. 62. Philadelphia, PA: SIAM, 1992. 